\title{
Campings matemáticos para el desarrollo del pensamiento crítico en estudiantes de básica secundaria en centros educativos rurales
}

\section{Mathematical campsites for the development of critical thinking in secondary basic students in rural educational centers.}

\author{
Dubian Peñaranda ${ }^{1}$ \\ Mawency Vergel Ortega² \\ Luisa Stella Paz Montes ${ }^{3}$
}

UFPS

\section{RESUMEN}

En la sociedad actual, donde los sujetos ameritan nuevas perspectivas de enseñanza, se requiere que los profesionales de la docencia se enfoquen en la planificación y puesta en práctica de estrategias y actividades didácticas dirigidas 1 Universidad Francisco de Paula Santander Cúcuta - Colombia, Orcid:0000-0001-8285-2968

Email: danipea21@hotmail.com

$2 \quad$ Universidad Francisco de Paula Santander Cúcuta - Colombia, Orcid: 0000-0002-8887-3441

Email: Iuisastellapm@ufps.edu.co-Ipazmontes@gmail.com a la orientación y adecuación de la enseñanza, sobre todo en áreas que impliquen complejidad para los estudiantes, como es el caso de la matemática. En tal sentido la investigación tiene como objetivo central "Evaluar la aplicación del camping matemático como estrategia en el desarrollo del pensamiento crítico en los estudiantes del CER. Nuestra Señora del Carmen". La investigación se realizó bajo el paradigma cualitativo, empleando como técnicas la entrevista y observación a través del guión de preguntas realizada a los estudiantes y docentes de la institución, las mismas que fueron 
analizados bajo la codificación abierta. Como hallazgos se obtuvo que las prácticas de los docentes se fundamentan en el tradicionalismo, como es la planificación y evaluación educativa. Ante esto se hizo necesaria la aplicación y sistematización de la propuesta de los campings matemáticos, los cuales obtuvieron gran recepción y resultados favorables por parte de los estudiantes.

\section{PALABRAS CLAVE:}

Pensamiento crítico, Matemáticas, Centros Educativos, Estudiantes.

\section{ABSTRACT}

In today's society, where subjects deserve new teaching perspectives, it is required that teaching professionals focus on the planning and implementation of teaching strategies and activities aimed at the orientation and adaptation of teaching, especially in areas that imply complexity for students, as is the case in mathematics. In this sense, the main objective of the research is "To evaluate the application of mathematical camping as a strategy in the development of critical thinking in CER students. Our Lady of Carmen". The research was carried out under the qualitative paradigm, using interview and observation as techniques through the script of questions made to the students and teachers of the institution, which were analyzed under open coding. As findings, it was obtained that the teachers' practices are based on traditionalism, such as educational planning and evaluation. Given this, it was necessary to apply and systematize the proposal of the mathematical campsites, which obtained great reception and favorable results from the students.

\section{KEY WORDS:}

Critical thinking, Mathematics, Educational Centers, Students.

\section{INTRODUCCIÓN}

Desde tiempos antiguos, la matemática ha contribuido a los fines de la educación y esta consideración lleva a pensar que se hace necesario planear y realizar actividades pedagógicas que estimulen positivamente el aprendizaje de los niños. Los lineamientos curriculares de matemáticas emanados por el Ministerio de Educación dan orientaciones sobre esta área. Las competencias matemáticas se requieren para el desarrollo de ambientes de aprendizaje en los cuales se puedan analizar situaciones problemáticas significativas $y$ comprensivas que permitan avanzar a niveles de competencia de más complejidad. Para que haya un conocimiento matemático se debe tener en cuenta el conocimiento conceptual y el conocimiento procedimental.

En este sentido, se hace necesario que el docente conozca y reconozca las debilidades, pero también las fortalezas de cada uno de los estudiantes, estos elementos no pueden dejarse de lado pues no se trata solo de repetir contenidos programáticos, sino de darles a conocer sistemas de valores que influyen en el aprendizaje de los sujetos, teniendo presente en cada momento, que los estudiantes son seres únicos entre sí, y son esas diferencias las que deben ser tomadas en cuenta por los docentes.

Es desde esta perspectiva, el docente a través de su quehacer didáctico está en el deber de crear vínculos entre lo aprendido y lo nuevo por adquirir, de tal manera que se resalte el valor social y universal de la matemática por su utilización en todos los campos del saber y vivir humano.

\section{DESCRIPCIÓN DE LA PROBLEMÁTICA}

Una de las principales funciones del sistema educativo en cualquier parte del mundo según los diferentes modelos curriculares, es promover una formación integral del sujeto y así lo expresa García (2006) quien considera que: 
en la sociedad del conocimiento, cada persona ha de asimilar una base de conocimientos rigurosos y estrategias eficaces; tiene que saber qué pensar y cómo actuar ante las situaciones relevantes a lo largo de la vida; hacerlo desde criterios razonables y susceptibles de crítica; ser sensible a las exigencias cambiantes de los contextos; desarrollar el pensamiento reflexivo, crítico y creativo. (p.2)

Las sociedades actuales requieren por parte de sus ciudadanos el dominio de habilidades en todas las dimensiones del ser (cognitivas, conductuales, emocionales, sociales...), las cuales serán adquiridas desde la temprana infancia y fortalecidas a lo largo de su vida. Estas competencias deben partir del dominio conceptual y estructural de contenidos para el desarrollo de esquemas mentales que serán luego tomados en consideración en el momento de la aprehensión de saberes. También deben inculcarse habilidades interpersonales, motivacionales hacia el logro de metas u objetivos propuestos. Los anteriores saberes, conocimientos, serán base parala sección procedimental de los acontecimientos propios en la construcción del saber.

En este sentido, la cultura de las instituciones educativas en la actualidad debe constituirse como el instrumento visible a través del cual, las escuelas llevan a cabo su trabajo en el campo educativo en la construcción del conocimiento para la formación de los ciudadanos; impregnada de toda una carga valórico-ideológica que conceptualiza el rol de la educación en una sociedad específica. Desde esta perspectiva, Parra (2014) señala que: La escuela, poseedora de una cultura escolar, comprende una forma de organización social, valores y normas que la forman, un concepto y manejo del poder, de la participación, de la solución de conflictos, reglas que rigen la violencia, relaciones entre maestros y alumnos, y disciplina. dentro y fuera del salón de clases. (p. 8)

A la institución educativa corresponde la tarea de iniciar las premisas sobre la construcción del conocimiento científico, organizando de manera sistemática la información recabada del contexto para lograr su adaptación al escenario en el cual se genera. Es a partir de ello, que se necesitan nuevas concepciones de las prácticas docentes, dejando de lado las divisiones o estructuras rígidas en los procesos didácticos. Hay que formar ciudadanos de forma significativa. Las implicaciones del conocimiento en la escuela se traducen en que ésta, es el lugar donde se produce; además de constituirse en el espacio donde se forman los ciudadanos.

El pensamiento crítico es un tema que no es novedoso, pues, como lo describe Ennis (1991), este concepto surge en Grecia en los siglos VII y VI a.C, debido al auge de la Filosofía y disciplinas afines como la lógica, la retórica y la dialéctica En el campo de la educación se inicia a mediados de los años sesenta, con tres dimensiones básicas: la lógica (juzgar, relacionar palabras con enunciados), el criterio (utilización de opiniones para juzgar enunciados) y la pragmática (comprensión del juicio y la decisión para construir y transformar el entorno).

El pensamiento crítico permite no sólo la aprehensión y construcción de conocimientos para otorgar significados, sino que permite además comprender el pensar del otro, respetar la opinión del otro y por ende establece relaciones de respeto en comunidad. De ello, que caracterizar una persona con un pensamiento crítico es complejo. Sin embargo, de acuerdo con Facione (2007) es una persona 
habitualmente inquisitiva; bien informada, que confía en la razón; de mente abierta; flexible; justa cuando se trata de evaluar; honesta cuando confronta sus sesgos personales; prudente al emitir juicios; dispuesta a reconsiderar y si es necesario a retractarse; clara respecto a los problemas o las situaciones que requieren la emisión de un juicio; ordenada cuando se enfrenta a situaciones complejas; diligente en la búsqueda de información relevante; razonable en la selección de criterios; enfocada en preguntar, indagar, investigar; persistente en la búsqueda de resultados tan precisos como las circunstancias y el problema o la situación lo permitan. (p. 21)

Campos (2007) señala que: "En la actualidad el sistema educativo colombiano, no es ajeno a la idea de que el estudio, comprensión y uso del pensamiento crítico no sólo es importante, sino también necesario en todos los niveles educativos" (p.9). Esta situación ha sido vista en los escenarios actuales, donde los niños demuestran especial interés por las acciones que le llevan a adoptar nuevas prácticas educativas como es el caso de la virtualidad, donde deben adaptarse a nuevas metodologías para lograr comprender lo que el docente está transmitiendo, y se exige de ellos posturas cognitivas con mayor nivel de complejidad, sumando a esto los sentimientos que le originan estar en condiciones de aislamiento por la emergencia sanitaria (Covid 19), suscitada actualmente, lo cual les ha llevado a adoptar un pensamiento crítico- analítico frente a la resolución de las problemáticas presentadas tanto en el campo personal como académico.

\section{FUNDAMENTACIÓN TEÓRICA}

\section{El pensamiento como proceso mental}

Referir a esta construcción conceptual es tomar en consideración la opinión de diferentes autores. Así, cuando Arredondo (2006), define al pensamiento como "la inteligencia interiorizada y se apoya no ya sobre la acción directa, sino sobre un simbolismo, sobre la evocación simbólica por medio del lenguaje o por imágenes

De acuerdo a las posiciones de los autores anteriores, el pensamiento no sólo se trata de las acciones sucedidas en el proceso mental, sino que se materializa en la medida que el sujeto refleja a través de sus acciones cotidianas, y que le permiten materializar su formación integral. De igual forma se puede afirmar que pensar es constante, razonar es más un acto de discernimiento elaborado.

Es de esta forma que estudiar el pensamiento es un tema de complejidad, donde se tiene que resaltar que el pensamiento es la capacidad o competencia general del ser humano para procesar información y construir conocimiento, combinando representaciones, operaciones y actitudes mentales, en forma automática, sistemática, creativa o crítica, para producir creencias y conocimientos, plantear problemas y buscar soluciones, tomar decisiones y comunicarse e interactuar con otros, y, establecer metas y medios para su logro.

\section{EL PENSAMIENTO CRÍTICO}

El pensamiento crítico constituye el nivel más alto del pensamiento del hombre, es el que permite su distinción de otra especie viva, desde este punto Para Ennis (1991), el pensamiento crítico se concibe como el pensamiento racional y reflexivo interesado en decidir qué hacer o creer. Es decir, por un lado, constituye un proceso cognitivo complejo de pensamiento que reconoce el predominio de la razón sobre las otras dimensiones del pensamiento. Su finalidad 
es reconocer aquello que es justo y aquello que es verdadero, es decir, el pensamiento de un ser humano racional.

De igual forma, Vallarini (2000), expone "llamamos pensamiento crítico a la capacidad del pensamiento para examinarse y evaluarse a sí mismo (el pensamiento propio o el de los otros), en términos de cinco dimensiones: lógica, sustantiva, contextual, dialógica y pragmática. La capacidad para el pensamiento crítico surge de la metacognición" Entonces, este nivel de pensamiento otorga al sujeto la capacidad de tomar decisiones entre diversas que puedan existir, autoevaluarse, y discernir de las acciones emprendidas con la finalidad de llevar a cabo las acciones y lograr así los objetivos propuestos. Este tipo de pensamiento proporciona la elaboración de puentes de conexión entre los presaberes con los nuevos que adquiera a través de la estimulación adecuada.

Al analizar las diferentes dimensiones que se manejan en el pensamiento crítico se tiene que en ellas se maneja la capacidad de relacionarse el propio individuo consigo mismo, con los demás bien sea pares o semejantes $y$ con el contexto que le rodea a través del cumplimiento de las normas correspondientes para su adaptación a la sociedad que pertenece. Además, una de las capacidades descritas se tiene la oportunidad que se desarrolla para llevar a la práctica lo aprendido desde una vertiente pragmática y significativa.

\section{ENSEÑANZA DE LA MATEMÁTICA.}

Para iniciar el estudio de la matemática se hace necesario revisar la génesis de su creación, teniendo para ello la exposición de Gómez (2006) cuando refiere que esta tiene sus orígenes desde el inicio del propio hombre cuando se encuentra la aparición de figuras geométricas en las pinturas y en los artículos de cerámica, también se sabe del uso del cálculo a través del apoyo de los dedos, por lo que se hayan registros en grupos de 5 y de 10 .

Posteriormente se tienen lugares como Egipto, China y Mesopotamia, como civilizaciones donde se utiliza de una manera más organizada la matemática. En Egipto, por ejemplo, se inició el proceso de multiplicación y división diferenciando las unidades, decenas y centenas. El pueblo Egipcio fue el primero en conseguir resolver problemas con números fraccionarios y aplicar su uso en diversos problemas que se les planteaban en su evolución como civilización.

Desde esta perspectiva, se hace prioritario estudiar la adaptación de la matemática en el campo educativo, teniendo que ésta se materializa con la implementación de la didáctica, siendo este tema muy complejo, sin embargo, Castro (2008) señala que "La mayor parte de los profesores comparten actualmente una concepción constructivista de las matemáticas y su aprendizaje. En dicha concepción, la actividad de los alumnos al resolver problemas se considera esencial para que éstos puedan construir el conocimiento". ( $p, 25)$.

Según Castro, los docentes deben manejar diferentes características de forma consciente, que contribuyan a la enseñanza y aprendizaje del área de matemática, reconociendo su importancia como actor mediador en el proceso didáctico, un docente actual tiene que manejar las herramientas necesarias para contextualizar los contenidos estipulados en el currículo escolar, los cuales deben responder a las necesidades e intereses de los estudiantes involucrados en el proceso. Pero, sobre todo, el docente está en el deber de adaptar las nuevas tecnologías en su actividad cotidiana, despertando así el interés por aprender de los estudiantes, además de incorporar en 
su quehacer pedagógico herramientas que enriquecen y facilitan sus labores.

\section{FUNDAMENTACIÓN METODOLÓGICA}

\section{Enfoque o paradigma}

Se tomó el enfoque interpretativo, o también llamado por autores como Yuni y Urbano cualitativo para la recolección y el análisis de la información que se recabó. Al respecto Yuni y Urbano (2005) señalan que este paradigma "obedece a la concepción filosófica de la que se nutre y que le provee los fundamentos acerca de lo real y las posibilidades de conocerlo". (p.107).

En este marco de fundamentación, Sandi (2003) afirma que las prácticas de enseñanza, como prácticas humanas no pueden ser abarcadas por explicaciones causales como las utilizadas para dar cuenta de los fenómenos naturales, sino que sólo pueden entenderse a la luz de los fines y razones que la impulsan, ya que en este caso no son otras que el bienestar de las personas. (p.58).

\section{Diseño de la Investigación}

El diseño utilizado fue de investigación de Campo, en virtud de recolectar la información directamente de la realidad y siendo propio del paradigma cualitativo. Al respecto la Universidad Pedagógica Experimental Libertador (2016) la define como: El análisis sistemático de problemas de la realidad, con el propósito bien sea de describirlos, interpretarlos, entender su naturaleza, para explicar sus causas y efectos, o predecir su ocurrencia, haciendo uso de métodos característicos de cualquiera de los paradigmas o enfoques de investigación conocidos. (p.16).

\section{Método de Investigación}

Se tomó la etnografía como modalidad cualitativa, con la finalidad de hacer un estudio exhaustivo de los eventos que ocurren en la vida del grupo referencial.

\section{Tipo de Investigación:}

Se optó por la Investigación Acción Colaboración -IAC- porque la población estudiada fue parte activa de la investigación al ser participe expresando las diferentes problemáticas y al aplicar los instrumentos ellos exponen sus propuestas de acuerdo a los objetivos de la investigación.

\section{Técnicas e Instrumentos de Recolección de Información}

Se hizo uso de la entrevista en profundidad, en la cual se utilizó el guion de preguntas abiertas. También se empleó la fotografía como técnica de verificación a través de la cual se tiene constancia de la ejecución de los campings matemáticos por cada uno de los estudiantes, desde sus hogares.

\section{ANÁLISIS Y PRESENTACIÓN DE RESULTADOS}

Se analizaron las opiniones dadas por los informantes, las cuales fueron divididas en tres (3) unidades temáticas que constituyen las ideas generales obtenidas a partir de los testimonios que surgieron de las entrevistas, los mismos dieron origen a tres (3) categorías. Cada una de estas divisiones se codificaron con las iniciales las unidades y categorías respectivamente, para plasmar textualmente cada testimonio de los sujetos y proceder a contrastarlos con la teoría existente que le den valor científico, realizándose así el proceso de triangulación. Posteriormente, se lleva a cabo el análisis de la información obtenida a través de las notas de campo, contrastando así lo dicho por los sujetos con lo realmente realizado por ellos.

\section{UNIDAD DE ANÁLISIS:}

\section{Conceptualizaciones (CONCEP)}

En atención a la perspectiva de los estudiantes entrevistados, la matemática es esencial en 
todos los ámbitos de la vida, cumpliendo así con el modelo contextual, donde se reconoce la significativa del área de matemática en la cotidianidad de los sujetos. Por tal razón los docentes deben considerar durante sus prácticas la programación de actividades que permita a los estudiantes establecer puentes de conexión entre el aprendizaje previo (contextual- empírico), con los nuevos que se están impartiendo en el momento presente, con la finalidad de lograr la significancia requerida para solucionar los problemas cotidianos de su realidad.

\section{UNIDAD DE ANÁLISIS: Actividades de Enseñanza (ACTENS)}

Dentro de las actividades del docente en el acto educativo, se tienen las propias del proceso de enseñanza- aprendizaje, como son la planificación, evaluación y selección de estrategias, entre otras, en opinión de los estudiantes éstas se caracterizan por ser de orden tradicional; y así se evidencia en los testimonios facilitados con respecto a la planeación, la cual se realiza al momento de desarrollar sus clases

En los testimonios facilitados por los estudiantes, la práctica pedagógica de los docentes se limita al seguimiento de pautas tradicionales. Donde se pone de manifiesto el centralismo en repetición de patrones, y explicación centrada en el conocimiento que los docentes poseen, también se refleja la figura del reforzamiento con la finalidad de materializar los conocimientos obtenidos por los estudiantes.

Es evidenciado que los docentes en estudio emplean diversidad de instrumentos de evaluación; pero también se nota que en una modalidad donde la participación de los actores tiene un carácter horizontal e igualitario entre los facilitadores y los participantes, se nota que prevalecen los instrumentos más inclinados a la medición que a la evaluación como tal.

\section{PROCESO DE TRIANGULACIÓN DE LOS HALLAZGOS}

Una vez presentada la información obtenida a través de las entrevistas, en contrastación con las evidencias fotográficas, se tiene que los docentes en la actualidad siguen la implementación de estrategias y práctica pedagógicas fundamentadas en paradigmas tradicionales, donde el estudiante se limita a reproducir el contenido basado en material escrito/textual, esto en lo respectivo a la planificación de la acción didáctica y a la evaluación.

También se evidencia que los sujetos son conscientes de la importancia de la matemática en la vida cotidiana por lo tanto su enseñanza debe fundamentarse en prácticas innovadoras, creativas, que estimulen el pensamiento crítico y la investigación continua, en espacios que estimulen en los estudiantes una actitud de recepción y empatía por las diferentes áreas académicas, sobre todo en la actualidad, que los estudiantes se encuentran realizando sus actividades desde los hogares.

En la actualidad se ha visto con mayor preponderancia el apoyo que existe entre contexto y escuela, en el proceso formativo de los estudiantes; siendo aquí donde se materializa la acción mediadora del docente en toda su amplitud. Como lo expresa García (2006): “EI maestro efectúa su mediación pedagógica en una ósmosis de vida y de trabajo con alumnos, familias y miembros de la comunidad" (p. 3). Se trata de una relación sistémica entre quien forma y quienes hacen parte del colectivo, pues en el proceso educativo no se educa únicamente al estudiante, sino que por cada estudiante que se está instruyendo y formando, incurre un grupo familiar que también está recibiendo de forma 
indirecta la realimentación bajo todas las esferas del conocimiento: (ser, hacer, conocer, convivir).

Desde esta perspectiva, Suárez (2005) expresa que el docente se convierte en: Fomentador de análisis, inductor de cambios, activador de búsqueda, motivador y facilitador de experiencias, suscitador de discusión y crítica, generador de hipótesis, planeador de problemas y alternativas, promotor y dinamizador de cultura, frente a un grupo estudiantil que piensa, crea, transforma, organiza y estructura conocimientos en un sistema personal y dinámico (p.65).

El rol del docente como mediador de las situaciones de aprendizaje mantiene un grado de complejidad, su labor trasciende la realizada en el espacio didáctico (aula), al desarrollo de contenidos, su función se centra en la relación interpersonal y humana con quienes les rodea, con la finalidad de consolidar en cada sujeto una vivencia y un aprendizaje de carácter significativo, adaptado a las condiciones reales de cada contexto y con la capacidad de resolver las diferentes situaciones que se puedan presentar en su cotidianidad.

Es un docente activo, innovador, que cada día se encuentra con un grupo de estudiantes con conocimiento propio (pre saberes) y que le permitirán a fortalecer su labor mediadora desde la esencia humana y profesional, mismos sujetos que están esperando del actuar del docente un intercambio fluido, respetuoso, empático, pero sobre todo que le conduzca al descubrimiento de nuevas experiencias.

\section{HALLAZGOS}

En esta sección, se presentan los hallazgos que se obtuvieron una vez se aplicó el diagnóstico, el mismo que se tomó en cuenta al momento de la elaboración y aplicación de la propuesta que se presenta en la siguiente sección. Con respecto al objetivo específico número 1: "Develar las estrategias que emplean los docentes de matemática para el desarrollo del pensamiento crítico en los estudiantes":

Se pudo constatar que las estrategias predominantes en la enseñanza de la matemática se inclinan hacia el enfoque tradicional, representadas por pruebas objetivas, instrumentos de orden cerrado, listas y escalas numéricas.

Aunque se da participación a los estudiantes, la misma es sólo con la finalidad de poder obtener un resultado de verificación de logros obtenidos.

Se tiene como principal estrategia y característica del enfoque tradicional el reforzamiento a través de la pregunta- respuesta, a partir de la cual el docente planifica las actividades a evaluar y desarrollar cada uno de los contenidos desarrollados.

En relación al objetivo específico número 2; "Analizar la actitud de los estudiantes frente a las metodologías empleadas por los docentes en la enseñanza de matemática" En los resultados obtenidos, se denotó una actitud favorable de parte de los estudiantes hacia el aprendizaje de la matemática, resaltando su utilidad en el desarrollo de las actividades cotidianas.

Los estudiantes manifiestan que como actividades de interés y con significancia tanto para la enseñanza como para la evaluación, es recomendable que el docente innove actividades, de preferencia con carácter lúdico, de tal manera que sea motivante, y más fácil de aprender los contenidos. 


\section{REFERENCIAS}

Arredondo, A. (2006). El pensamiento: una definición interconductual. [Trabajo en línea]. Disponible:http://sisbib.unmsm.edu. pe/bvrevistas/investigacion_psicologia/ v03 n1/pdf/a02v3n1.pdf

Campos, A. (2007). Pensamiento crítico (1 $1^{\mathrm{a}} \mathrm{Ed}$.). Bogotá: Editorial Magisterio.

Castro, E. (2008). Didáctica de la matemática en la Educación Primaria. Madrid:Editorial Síntesis.

Ennis,R.(1991).

CriticalThinking:Astreamlinedconception. TeachingPhilosophy.

Facione, P. (2007). ¿Qué es el pensamiento crítico y por qué es importante? [Documento en línea]. Disponible: https://www. researchgate.net/publication/237469559 Pensamiento_Critico_Que_es_y_por que es importante

García García, E. (2006). Las competencias del profesor en la sociedad del conocimiento. En R. Mejía (Coord.). Educación, Globalización y Desarrollo Humano. Santo Domingo, RD: Editora Buho.

Gómez, De Corte, E. (2006). Creencias de los estudiantes de matemáticas. La influencia del contexto de clase. Enseñanza de las Ciencias.

Latorre, Arnal y Rincón. (1996). Bases Metodológicas de la Investigación Cualitativa. Armenia- Colombia. Ediciones Experiencia

Parra, K. (2014). El docente y el uso de la mediación en los procesos de enseñanza y aprendizaje. [Artículo en línea]. Universidad Pedagógica Experimental LibertadorCaracas. Disponible: hrome-extension:// ohfgljdgelakfkefopgklcohadegdpjf/ h t t p s: / / w w w. reda I y c.org / pdf/3761/376140398009.pdf

Sandín, M. (2003). Investigación cualitativa en educación: Fundamentos y tradiciones. Madrid: McGraw-Hill

Suárez, R. (2005). La educación: teorías educativas. Estrategias de enseñanza y aprendizaje. Madrid: Trillas

Universidad Pedagógica Experimental Libertador [UPEL] (2016). Manual de Trabajos de Grado de Especialización y Maestrías y tesis doctorales. Caracas.

Villarini (2000). Teoría y Pedagogía del pensamiento crítico

Yuni y Urbano (2005). Técnicas para investigar. 